\title{
Correlation Between Maternal and Neonatal Vitamin B12 Serum Levels in Fullterm Neonates with Physiologic Jaundice
}

Reem A. Abdel Aziz ${ }^{1^{*}}$ and Mostafa A. Abu Elela ${ }^{2}$

${ }^{1}$ Department of Pediatrics, Faculty of Medicine, Minia University, Minya, 11432, Egypt

${ }^{2}$ Department of Clinical-pathology, Faculty of Medicine, Minia University, Minya, 11432, Egypt

\section{Abstract}

Introduction: Vitamin B12 (vitB12)deficiency causes failure of red blood cells (RBC) maturation leading to cell lysis causing excess heme production resulting in indirect hyperbilirubinemia.

Purpose: We aimed toassess vitB12 serum levels in neonates with jaundice and compare these levels with that of apparently healthy neonates and detect the impact of the maternal vitB12 on both vitB12 and bilirubin of their neonates.

Patients and methods: Complete blood count $(\mathrm{CBC}), \mathrm{C}$ - reactive protein $(\mathrm{CRP})$, and VitB12 were assessed in both groups; 60 full term neonates with jaundice (Group I) and 60 full term controls. VitB12 levels were assessed for the mothers of all included neonates.

Results: VitB12 in jaundice neonates were significantly lower than that of controls. Maternal vitB12 was positively correlated with neonatal vitB12 and negatively correlated with neonatal total serum bilirubin levels (TSB).

Conclusion: Low maternal vitB12 affects both vitB12 and bilirubin levels of their neonates.

\section{Introduction}

Physiologic jaundice is caused by a combination of increased bilirubin production (secondary to accelerated destruction of erythrocytes and decreased excretory capacity secondary to low levels of ligandin in hepatocytes) and low activity of the bilirubinconjugation enzyme uridine diphospho glucuronyltransferase (UDPGT) [1].

VitB12 is essential for maturation and proliferation of RBCs [2]. Deficiency of VitB12 causes failure of RBC maturation leading to cell lysis which causes excess heme production resulting in indirect hyperbilirubinemia [3].

Hemolysis in patients with vitB12 deficiency is a well-recognized phenomenon and it is due to intramedullary destruction of erythrocytes (ineffective erythropoiesis). Homocysteine increases the risk of hemolysis in vitB12 deficiency and there is a high frequency of vitB12 deficiency in asymptomatic patients with homozygous methylenetetrahydrofolate reductase (MTHFR) C677T mutation, a known cause of hyperhomocysteinemia [4].

Patients demonstrated complete resolution of hemolysis with simultaneous normalization of serum homocysteine levels after vitB12 treatments [5].

\section{Aim of the Work}

The aim of this study is to assess vitB12 serum levels in neonates with physiologic jaundice, compare them with those of apparently healthy neonates and detect the impact of maternal serum vitB12 on both vitB12 and serum bilirubin of their neonates.

\section{Patients and Methods}

One hundred twenty full term neonates and their mothers were enrolled from Neonatal Care Unit and Obstetric Department of Minia University Hospital, Minia, Egypt in the period from February to December 2017.
They were divided into 2 groups:

Group 1: 60 full term neonates with indirect hyperbilirubinemia (34 females and 26 males) aged 3-7 days.

Group 2: 60 apparently healthy full term neonates as control group (29 males and 31 females) of matched age, weight and sex.

All neonates were subjected to complete history taking; the onset of appearance of jaundice, gestational age, mode of delivery and history suggestive for sepsis (bad general condition, fever, poor suckling) or suggestive for obstructive jaundice(dark urine and pale stool).

All neonates were subjected to complete history taking; the onset of appearance of jaundice, gestational age, mode of delivery and history suggestive for sepsis (bad general condition, fever, poor suckling) or suggestive for obstructive jaundice(dark urine and pale stool).

Clinical examination was done; general condition, pallor, fever, vital signs (temperature, respiratory rate, heart rate), reflexes, weight, and systemic examination for organomegaly.

All patients were chosen according to the following inclusion and exclusion criteria; Inclusion criteria included: 3-7 days aged full term neonates, good general condition (good moro and suckling), laboratory investigations; TSB more than $5 \mathrm{mg} / \mathrm{dl}$ with direct bilirubin $<20 \%$, CBC: normal $\mathrm{Hb}$ levels, platelet and WBCs count and no other signs suggestive for infection and -ve CRP.

"Corresponding Author: Dr. Reem A. Abdel Aziz, Department of Pediatrics, Faculty of Medicine, Minia University, Minya, 11432, Egypt; E-mail: reemabdelsalam3@gmail.com

Citation: Aziz RAA, Elela MAA (2018) Correlation Between Maternal and Neonatal Vitamin B12 Serum Levels in Fullterm Neonates with Physiologic Jaundice. Int J Pediatr Neonat Care 3: 137. doi: https://doi.org/10.15344/24552364/2018/137

Copyright: () 2018 Aziz et al. This is an open-access article distributed under the terms of the Creative Commons Attribution License, which permits unrestricted use, distribution, and reproduction in any medium, provided the original author and source are credited. 
Citation: Aziz RAA, Elela MAA (2018) Correlation Between Maternal and Neonatal Vitamin B12 Serum Levels in Fullterm Neonates with Physiologic Jaundice. Int J Pediatr Neonat Care 3: 137. doi: https://doi.org/10.15344/2455-2364/2018/137

Page 2 of 5

Neonates with gestational age less than 37 weeks and more than 42 weeks, ages less than 3 days and more than 7 days, neonates with bad general condition (absent or weak moro or suckling), organomegaly, neonates with $\mathrm{Rh}$ or $\mathrm{ABO}$ incompatibility, Glucose 6- phosphate Dehydrogenase Deficiency (G6PD), hypothyroidism, polycythemia, +ve CRP, Direct bilirubin $>20 \%$, CBC: signs of infection (thrombocytopenia, leukopenia leukocytosis, differential count of WBCs shows shift to lift) were excluded from our study.

\section{Laboratory investigations}

Five $\mathrm{ml}$ of venous blood were obtained from each neonate under sterile venipuncture and divided as follow:

One $\mathrm{ml}$ was added to EDTA containing tube for performing $\mathrm{CBC}$.

Four $\mathrm{ml}$ were put on a plain plastic tube, incubated for 20 minutes at $37^{\circ} \mathrm{c}$ and centrifuged at 3000 r.p.m for 10 minutes. The serum aliquot was divided into two parts; one for serum bilirubin and CRP while the remaining part stored at $-20^{\circ} \mathrm{C}$ in Eppendorf cups until processing serum vitB12.

\section{CBC assessment}

Done by automated cell counter symex KX21N (JAPAN).

\section{Serum bilirubin}

Done by automated chemical analyzer Konelab 60 i(FILAND).

\section{CRP}

Done by qualitative and semiquantitated determination latex agglutination spin react (SPAIN) [6,7].

\section{Serum VitB12 level}

By quantitative method using ELISA kit. Glory Science Co, Ltd (CHINA, HONG KONG).

VitB12 levels were also measured for the mothers of all included neonates.

\section{Statistical methods}

The collected data were coded, tabulated, and statistically analyzed using SPSS program (Statistical Package for Social Sciences) software version 24.

Descriptive statistics were done for parametric quantitative data by mean, standard deviation and minimum \& maximum of the range, while they were done for categorical data by number and percentage.

Analyses were done for parametric quantitative data between the two groups using independent samples T test.

Analyses were done for qualitative data using Chi square test.

Correlation between two quantitative variables was done by using Pearson's correlation coefficient. Weak $(\mathrm{r}=0-0.24)$, fair $(\mathrm{r}=0.25-0.49)$, moderate $(\mathrm{r}=0.5-0.74)$, strong $(\mathrm{r}=0.75-1)$.
Simple linear regression analysis was used for prediction of vitB12 level.

ROC curve analysis of vitB12 was used for prediction of jaundice severity. The level of significance was taken at $(\mathrm{P}$ value $<0.05)$.

\section{Results}

There was no significant difference between the two studied groups regarding the demographic and the laboratory data as shown in tables 1 and 2. There was no significant difference between the two groups regarding the levels of $\mathrm{Hb}$, platelets, $\mathrm{WBC}$ and neutrophils. Also, both groups had -ve CRP.

\begin{tabular}{|c|c|c|c|}
\hline & $\begin{array}{l}\text { Control } \\
(n=60)\end{array}$ & $\begin{array}{l}\text { Patient } \\
(n=60)\end{array}$ & $P$ value \\
\hline \multicolumn{4}{|l|}{ Age } \\
\hline Range & $(3-7)$ & $(3-7)$ & \multirow[t]{2}{*}{0.413} \\
\hline Mean \pm SD & $4.3 \pm 1.3$ & $4.4 \pm 1.1$ & \\
\hline \multicolumn{4}{|l|}{ Sex } \\
\hline Male & $29(48.3 \%)$ & $26(43.3 \%)$ & \multirow[t]{2}{*}{0.583} \\
\hline Female & $31(51.7 \%)$ & $34(56.7 \%)$ & \\
\hline \multicolumn{4}{|c|}{ Mode of delivery } \\
\hline SVD & $22(36.7 \%)$ & $24(40 \%)$ & \multirow[t]{2}{*}{0.707} \\
\hline CS & $38(63.3 \%)$ & $36(60 \%)$ & \\
\hline \multicolumn{4}{|c|}{ Gestational age } \\
\hline Range & $(37-41)$ & $(37-41)$ & \multirow[t]{2}{*}{0.930} \\
\hline Mean \pm SD & $38.4 \pm 1$ & $38.4 \pm 1$ & \\
\hline \multicolumn{4}{|l|}{ Weight } \\
\hline Range & $(2.5-3.7)$ & $(2.5-3.6)$ & \multirow[t]{2}{*}{0.413} \\
\hline Mean \pm SD & $3.1 \pm 0.4$ & $3.1 \pm 0.3$ & \\
\hline
\end{tabular}

Table 1: Demographic data of the studied groups.

Independent samples $\mathrm{T}$ test for parametric data between the two groups

Chi square test for qualitative data between the two groups

*: Significant level at $\mathrm{P}$ value $<0.05$.

\begin{tabular}{|c|c|c|c|}
\hline & $\begin{array}{l}\text { Control } \\
(n=60)\end{array}$ & $\begin{array}{l}\text { Patients } \\
(n=60)\end{array}$ & $P$ value \\
\hline \multicolumn{4}{|l|}{$\mathrm{Hb}$} \\
\hline Range & $(14-18.9)$ & $(14.3-19)$ & \multirow[t]{2}{*}{0.696} \\
\hline Mean \pm SD & $16.4 \pm 1.4$ & $16.5 \pm 1.4$ & \\
\hline \multicolumn{4}{|l|}{ Platelets } \\
\hline Range & $(158-451)$ & $(150-448)$ & \multirow[t]{2}{*}{0.767} \\
\hline Mean \pm SD & $293 \pm 79.7$ & $288.4 \pm 91.5$ & \\
\hline \multicolumn{4}{|l|}{ WBCs } \\
\hline Range & $(4.5-10.9)$ & $(4-11)$ & \multirow[t]{2}{*}{0.650} \\
\hline Mean \pm SD & $7.5 \pm 1.8$ & $7.7 \pm 2$ & \\
\hline \multicolumn{4}{|l|}{ Neutrophils } \\
\hline Range & $(48-67.7)$ & $(48.5-65.1)$ & \multirow[t]{2}{*}{0.606} \\
\hline Mean \pm SD & $55.7 \pm 5.2$ & $55.2 \pm 4.7$ & \\
\hline CRP & -ve & -ve & \\
\hline
\end{tabular}

Table 2: CBC, CRP findings in both groups. 
Citation: Aziz RAA, Elela MAA (2018) Correlation Between Maternal and Neonatal Vitamin B12 Serum Levels in Fullterm Neonates with Physiologic Jaundice. Int J Pediatr Neonat Care 3: 137. doi: https://doi.org/10.15344/2455-2364/2018/137

Page 3 of 5

TSB levels were significantly higher in patients than controls $\left(15.7 \pm 2.9\right.$ and $3 \pm 0.8$ respectively, $\left.\mathrm{p}<0.001^{\star}\right)$. On contrast, neonatal vitB12 levels were significantly lower in patients than in controls $\left(226.4 \pm 25.5\right.$ and $421.8 \pm 81.3$ respectively, $\left.\mathrm{p}<0.001^{\star}\right)$. VitB12 levels were also significantly lower in the patients' mothers than that of controls (158.1 \pm 16 versus $217.7 \pm 24$ ) (Table 3 ).

Neonatal vitB12 levels were negatively correlated with their serum bilirubin levels $\left(\mathrm{r}=-0.873, \mathrm{p}<0.001^{\star}\right)($ Table $4 \mathrm{~A})$.

Maternal vitB12 levels were negatively correlated with neonatal serum bilirubin levels and positively correlated with neonatal vitB12 levels $\left(\mathrm{r}=-0.923, \mathrm{p}<0.001^{\star}\right.$ and $\mathrm{r}=0,828, \mathrm{p}<0.001^{*}$ respectively) (Table 4B).

By using Linear Simple Regression analysis, we can predict serum vitB12 level from TSB level by the following equation:

VitB12 $=345.251+(-7.572 \times$ TSB $)($ Table 5$)$.

\begin{tabular}{|c|c|c|c|}
\hline & $\begin{array}{l}\text { Control } \\
(n=60)\end{array}$ & $\begin{array}{l}\text { Patients } \\
(n=60)\end{array}$ & $\mathrm{P}$ value \\
\hline \multicolumn{4}{|c|}{ Neonatal TSB } \\
\hline Range & $(1.2-4.9)$ & $(9-20.5)$ & \multirow[t]{2}{*}{$<0.001^{*}$} \\
\hline Mean \pm SD & $3 \pm 0.8$ & $15.7 \pm 2.9$ & \\
\hline \multicolumn{4}{|c|}{ Neonatal VitB12 } \\
\hline Range & $(270-590)$ & $(160-263)$ & \multirow[t]{2}{*}{$<0.001^{*}$} \\
\hline Mean \pm SD & $421.8 \pm 81.3$ & $226.4 \pm 25.5$ & \\
\hline \multicolumn{4}{|c|}{ Maternal Vit B12 } \\
\hline Range & $(176-250)$ & $(130-190)$ & \multirow[t]{2}{*}{$<0.001^{*}$} \\
\hline Mean \pm SD & $217.7 \pm 24$ & $158.1 \pm 16$ & \\
\hline
\end{tabular}

Table 3: comparison between Neonatal TSB, VitB12 and maternal VitB12 between both groups.

\begin{tabular}{|l|l|l|}
\hline \multirow{2}{*}{ Patient group } & \multicolumn{2}{|c|}{ Neonatal VitB12 } \\
\cline { 2 - 3 } & $\mathrm{R}$ & P value \\
\hline Age & 0.040 & 0761 \\
\hline Gestational age & 0.182 & 0.164 \\
\hline Weight & -0.071 & 0.588 \\
\hline TSB & -0.873 & $<0.001^{\star}$ \\
\hline
\end{tabular}

Table 4A: correlation between neonatal vitB12 and other parameters.

\begin{tabular}{|l|l|l|}
\hline \multirow{2}{*}{} & \multicolumn{2}{|c|}{ Maternal vit B12 } \\
\cline { 2 - 3 } & $\mathrm{r}$ & P value \\
\hline Neonatal TSB & -0.923 & $<0.001^{\star}$ \\
\hline Neonatal VitB12 & 0.828 & $<0.001^{\star}$ \\
\hline
\end{tabular}

Table 4B: correlation between maternal vitB12 and other parameters.

\begin{tabular}{|l|l|l|l|l|l|}
\hline & $\mathrm{B}$ & P value & $\mathrm{R}$ & $\mathrm{R}^{2}$ & $\begin{array}{l}\text { Regression } \\
\text { equation }\end{array}$ \\
\hline Constant & 345.251 & $<0.001^{*}$ & 0.873 & 0.762 & $\begin{array}{l}\mathrm{VitB}_{12}=345.251 \\
+(-7.572 \times \mathrm{TSB})\end{array}$ \\
\hline TSB & -7.572 & $<0.001^{*}$ & & & \\
\hline
\end{tabular}

Table 5: Linear simple regression analysis for prediction of $\mathrm{VitB}_{12}$ from TSB level.
By using the Roc curve analysis of neonatal jaundice for prediction of VitB12 severity, we could predict that vitB12 serum level is $\leq 249$ when TSB exceeds $14 \mathrm{mg} / \mathrm{dl}$ (sensitivity $97.7 \%$, specificity $87.5 \%$, and accuracy 95\%) (Figure 1).

\section{Discussion}

Jaundice is the most common condition that requires medical attention and hospital admission in newborns. In most infants, unconjugated hyperbilirubinemia reflects a normal transitional phenomenon. However, in some infants, serum bilirubin levels may rise excessively, which can be a cause for concern because unconjugated bilirubin is neurotoxic causing lifelong neurologic sequelae in infants who survive [8].

VitB12 (Cobalamin) is a water-soluble vitamin that is derived from animal products. Intrinsic factor (IF) is a glycoprotein that is produced by the parietal cells in the stomach and it is necessary for the absorption of vitB12 in the terminal ileum. Once absorbed, vitB12 is used as a cofactor for enzymes that are involved in the synthesis of DNA, fatty acids, and myelin. As a result, vitB12 deficiency can lead to hematologic and neurologic symptoms [9].

The prevalence of low cobalamin status is high especially in lowincome areas. Folate and cobalamin play interdependent roles in one-carbon metabolism (a group of biochemical reactions that have a special set of enzymes and coenzymes. They are involved in amino acid metabolism and also play roles in nucleotide metabolism) [10].

Although folic acid supplementation during early pregnancy is widely recommended, cobalamin supplementation during pregnancy and lactation has received little attention. Furthermore, the intake recommendations for pregnant and lactating women and in early life need reevaluation in the light of newly available evidence in the field [11].

The aim of our study is to assess the serum level of vitB12 in neonates with physiologic jaundice and compare them with those of apparently healthy neonates. On the other hand, we measured vitB12 serum levels of the mothers of these neonates to study the effect of maternal vitB12 on both neonatal vitB12 and serum bilirubin levels.

Sixty neonates (group I) with physiologic jaundice (after exclusion of causes of pathologic jaundice) and 60 apparently healthy neonates (group II) as controls were included in our study, mothers of all neonates of both groups were also included.

There was no statistical difference between both groups regarding demographic data. Also, there was no difference regarding Hb, WBCs, platelets, neutrophils levels and CRP between the groups, while total bilirubin levels were significantly higher in patients than in controls.

By measuring vitB12 levels in both groups, we found that vitB12 levels in neonates with jaundice were significantly lower than that of controls.

The mechanism of hemolysis is ineffective erythropoiesis, immature erythrocytes are lysed within the bone marrow, resulting in the release of excess of biliverdin, which is converted to indirect bilirubin. VitB12 deficiency is a correctable cause for hemolysis [12]. 


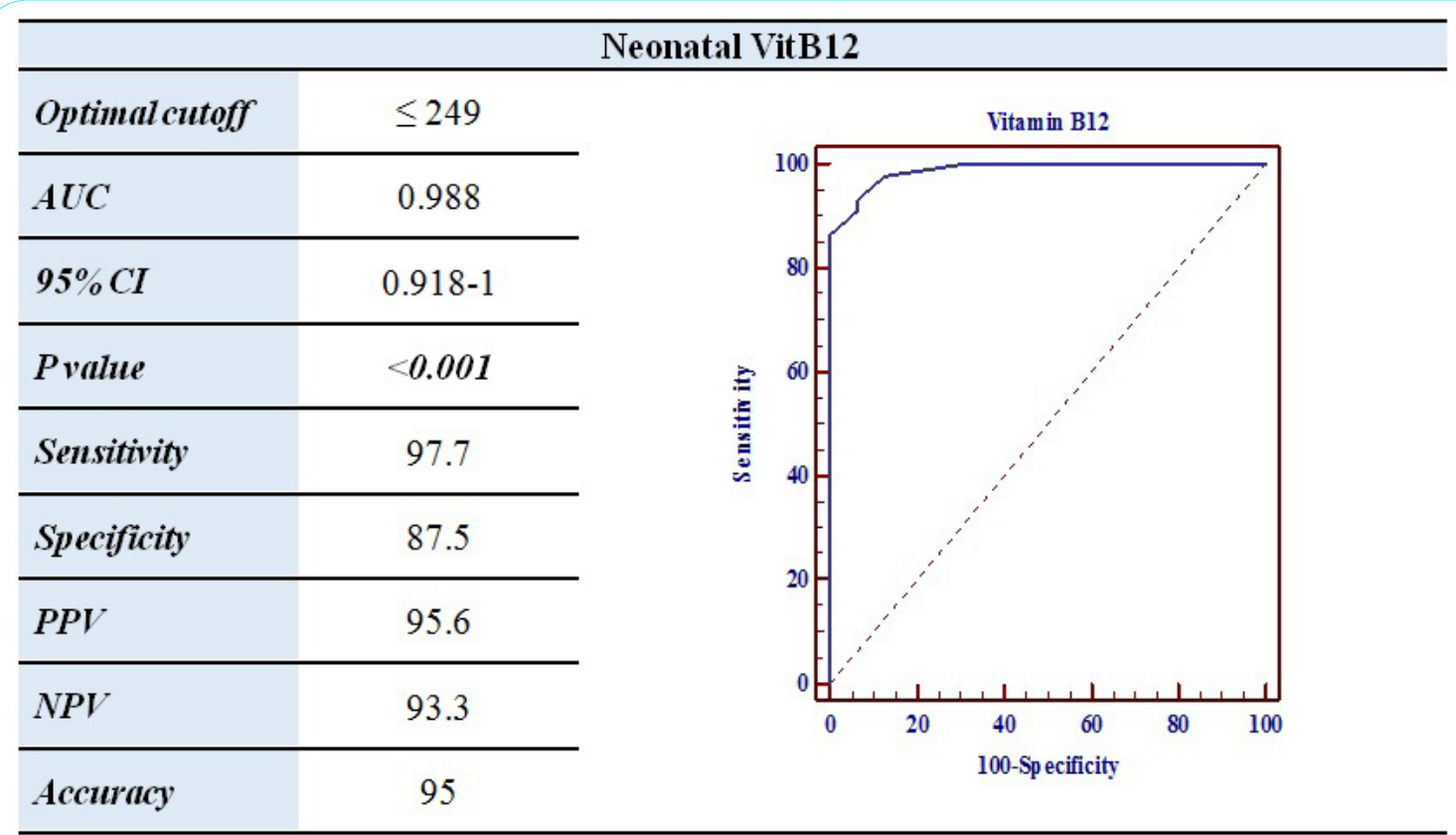

Figure 1: ROC curve analysis of neonatal jaundice for prediction of VitB12 severity (TSB > 14).

AUC: area under curve; CI: Confidence interval; PPV: positive predective value; NPV: negative predective value

Intramedullary destruction or hemolysis of fragile and abnormal red blood cell precursors is the result of ineffective erythropoiesis secondary to defective DNA and cell maturation. The hemolytic picture may resemble microangiopathic hemolytic anemia. If such condition persisted, it may deplete iron storage and lead to concomitant iron deficiency anemia [13].

In newborns, the functional capacity of liver is only about $1 \%$ of that of the adult. RBC lysis and low functional capacity of liver result in inefficient clearance of bilirubin and so indirect hyperbilirubinemia develops [14].

Our result is in agreement with that of Eroglu et al. [3] who found that the mean vitB12 level in patients was $119.9 \pm 43.9 \mathrm{ng} / \mathrm{L}$ (42.35 $178 \mathrm{ng} / \mathrm{L})$ while in controls was $286.17 \pm 97.43 \mathrm{ng} / \mathrm{L}(207.90-624.10$ $\mathrm{ng} / \mathrm{L})$. They also stated that low vitB12 level has been observed as a risk factor in neonatal hyperbilirubinemia.

In our study, vitB12 levels in mothers of neonates with jaundice were lower than their levels in mothers of controls and the maternal vitB12 level was positively correlated with neonatal vitB12.

This result was in agreement with that of Obeid et al., 2017 [11] who stated that women who began pregnancy with depleted stores (low or very low plasma cobalamin) will give birth to depleted infants who are likely to develop deficiency symptoms during the first few weeks or months postpartum. Newly ingested cobalamin during pregnancy and lactation is transferred to the child and is not likely to correct cobalamin status in depleted women [11].

VitB12 deficiency is more common in pregnant, lactating women and their young children in developing countries due to malabsorption which is exacerbated by low dietary intake of the vitamin $[15,16]$.
VitB12 levels fall progressively throughout pregnancy in most healthy women. This decline begins in the first trimester and levels may drop to 50 percent of pre-pregnancy values by the third trimester [17]. That is why in our study, maternal vitB12 levels in both groups were lower than the normal adult levels.

The normal physiologic changes during pregnancy contribute to the fall in vitB12 levels. Hemodilution (in which the plasma volume increases by 40 to 50 percent) lowers the vitamin concentration. Because the kidneys filter blood at a higher rate during pregnancy, more vitB12 will be lost in urine. VitB12 is actively transferred across the placenta to the developing fetus. VitB12 levels in umbilical cord and fetal blood are twice as high as in maternal blood, indicating preferential delivery to the fetus [17].

This is the first study showing that maternal vitB12 was negatively correlated with neonatal hyperbilirubinemia.

There is an evidence of increased vitB12 absorption during pregnancy, with the newly absorbed vitB12 being more important to placental transport than maternal liver stores. So that, lack of vitB12 rich diet of the mother may cause vitB12deficiency in newborn [3]. So, it is recommended that vitB12 supplement is necessary to avoid both neonatal vitB12 deficiency and hyperbilirubinemia.

We could also recommend vitB12 intake for neonates with exaggerated physiologic jaundice.

\section{Acknowledgments}

The authors like to thank all patients and their families for their involvement in this study. We also like to thank the Obstetric Department especially assistant professor M. Hosni for his great help in collecting data. 
Citation: Aziz RAA, Elela MAA (2018) Correlation Between Maternal and Neonatal Vitamin B12 Serum Levels in Fullterm Neonates with Physiologic Jaundice. Int J Pediatr Neonat Care 3: 137. doi: https://doi.org/10.15344/2455-2364/2018/137

Page 5 of 5

\section{Authors' Contributions}

Reem A. Abdel Aziz and Mostafa A. Abu Elela conceived the study, carried out its designing, coordinated the implementation, helped to perform the statistical analysis and drafted the manuscript. RA designed the study, participated in the analysis and interpretation of data and revised the statistics and final draft of the manuscript. MA was responsible for interpretation of laboratory data of patients and revision of the manuscript. All authors read and approved the final manuscript.

\section{Conflicting Interests}

The authors declare that there are no potential conflicts of interest with respect to the research, authorship, and/or publication of this article.

\section{References}

1. Memon N, Weinberger BI, Hegyi T, Aleksunes LM (2015) Inherited disorders of bilirubin clearance. Pediatr Res 79: 378-386.

2. Khanduri U, Sharma A (2007) Megaloblastic anemia: prevalence and causative factors. Natl Med J India 20: 172-175.

3. Eroglu N, Kandur Y, Kalay S, Kalay Z, Guney O, et al. (2015) Neonatal hyperbilirubinemia in a Turkish cohort: association of vitamin B12. J Clin Med Res. 7: 556-559.

4. Acharya U, Gau JT, Horvath W, Ventura P, Hsueh CT, et al. (2008) Hemolysis and hyperhomocysteinemia caused by cobalamin deficiency: three case reports and review of the literature. J Hematol Oncol 1: 26.

5. Katakam PK, Hegde AP, Venkataramaiahyappa M (2018) Vitamin B12 deficiency: unusual cause of jaundice in an adolescent. BMJ Case Rep.

6. Vaishnavichetana (1996) CRP kits. Immunology and infectious diseases 6:139-144.

7. Hokamayoshity (1987) CRP kits. Journal of clinical laboratory status 1:1527.

8. Mitra S, Rennie J (2017) Neonatal jaundice: aetiology, diagnosis and treatment. Br J Hosp Med (Lond) 78: 699-704.

9. Ankar A, Bhimji SS (2017) Vitamin, B12 (Cobalamin), Deficiency. StatPearls. Treasure Island (FL): StatPearls Publishing.

10. Ducker GS, Rabinowitz JD (2017) One-Carbon Metabolism in Health and Disease. Cell Metabolism 25: 27-42.

11. Obeid R, Murphy M, Solé-Navais P, Yajnik C (2017) Cobalamin Status from Pregnancy to Early Childhood: Lessons from Global Experience. AdvNutr 8: 971-979.

12. Aslinia F, Mazza JJ, Yale SH (2006) Megaloblastic anemia and other causes of macrocytosis. Clin Med Res 4: 236-241.

13. Stabler SP (2013) Clinical practice. Vitamin B12 deficiency. The New England Journal of Medicine. 368: 149-160.

14. Guilland JC, Aimone-Gastin I (2013) [Vitamin B12 (cobalamin)]. Rev Prat 6: $1085-10890$.

15. Allen LH (1994) Vitamin B12 metabolism and status during pregnancy, lactation and infancy. Adv Exp Med Biol 352: 173-186.

16. Casterline JE, Allen LH, Ruel MT (1997) Vitamin B12 deficiency is very prevalent in lactating Guatemalan women and their infants at three months postpartum. J Nutr 127: 1966-1972.

17. Pregnancy \& B12 Level. LIVESTRONG.COM. 